\title{
The genus, the Frobenius number, and the pseudo-Frobenius numbers of numerical semigroups of type two
}

\author{
Aureliano M. Robles-Pérez*† and José Carlos Rosales*‡
}

\begin{abstract}
In this paper we study some questions on numerical semigroups of type two. On the one hand, we investigate the relation between the genus and the Frobenius number. On the other hand, for two fixed positive integers $g_{1}, g_{2}$, we give necessary and sufficient conditions in order to have a numerical semigroup $S$ such that $\left\{g_{1}, g_{2}\right\}$ is the set of its pseudo-Frobenius numbers and, moreover, we build explicitly families of such numerical semigroups.
\end{abstract}

Keywords: Frobenius number, pseudo-Frobenius number, numerical semigroups, genus, type.

2010 AMS Classification: 11D07, 20M14, 13H10.

\section{Introduction}

Let $\mathbb{Z}$ and $\mathbb{N}$ be the set of integers and non-negative integers, respectively. A numerical semigroup is a subset $S$ of $\mathbb{N}$ that is closed under addition, $0 \in S$, and $\mathbb{N} \backslash S$ is finite.

If $A$ is a non-empty subset of $\mathbb{N}$, we denote by $\langle A\rangle$ the submonoid of $(\mathbb{N},+)$ generated by $A$, that is,

$$
\langle A\rangle=\left\{\lambda_{1} a_{1}+\cdots+\lambda_{n} a_{n} \mid n \in \mathbb{N} \backslash\{0\}, a_{1}, \ldots, a_{n} \in A, \lambda_{1}, \ldots, \lambda_{n} \in \mathbb{N}\right\} .
$$

It is well known that $\langle A\rangle$ is a numerical semigroup if and only if $\operatorname{gcd}(A)=1$. Moreover, every numerical semigroup $S$ is finitely generated and, therefore, there exists a finite subset $A$ of $S$ such that $S=\langle A\rangle$. In addition, if no proper subset of $A$ generates $S$, then we say that $A$ is a minimal system of generators of $S$. In fact, every numerical semigroup $S$ has a unique minimal system of generators. The cardinality of such a minimal system is called the embedding dimension of $S$ and is denoted by e $(S)$.

From the above paragraph, we deduce that the studying of numerical semigroups is related to the studying of the non-negative integer solutions of linear equations with coefficients in $\mathbb{N}$. Thereby, this is a classic problem that has been widely studied in the literature (see for instance [3, 4, 6, 7, 10]). Along this line, two invariants of a numerical semigroups $S$ play a particularly relevant role. The first one is the greatest integer that does not belong to $S$, the so-called Frobenius number of $S$, denoted by $\mathrm{F}(S)$. The second is the cardinality of $\mathbb{N} \backslash S$, the so-called genus of $S$, denoted by $\mathrm{g}(S)$.

The Frobenius problem (see [14]) consists of finding formulas for the Frobenius number and the genus of a numerical semigroup in terms of its minimal system of generators. This problem was solved by Sylvester and Curran Sharp (see [23, 24, 25]) when the embedding dimension is equal to two. However, it is an open problem for the case of embedding dimension $e \geq 3$. To be precise, in [5] it was shown that it is impossible to find a polynomial formula (that is, a finite set of polynomials) that computes the Frobenius number if $e=3$. In spite of this, some algorithms and partial answers to this question are known (see [9, 11, 13, 15, 16, 17, 18,).

The concept of type of a numerical semigroup $S$ was introduced in [8] as the cardinality of the set $\{x \in$ $\mathbb{Z} \backslash S \mid x+s \in S$ for all $s \in S \backslash\{0\}\}$ and was denoted by $\mathrm{t}(S)$. Following the notation of [20, we will denote such a set by $\operatorname{PF}(S)$ and its elements will be called pseudo-Frobenius numbers of $S$.

In 8 it was shown that all numerical semigroups with embedding dimension two have type equal to one, and that all numerical semigroups with embedding dimension three have type equal to one or two. Moreover,

\footnotetext{
*Both of the authors are supported by FQM-343 (Junta de Andalucía), MTM2010-15595 (MICINN, Spain), and FEDER funds. The second author is also partially supported by Junta de Andalucía/Feder grant number FQM-5849.

${ }^{\dagger}$ Departamento de Matemática Aplicada, Universidad de Granada, 18071-Granada, Spain.

E-mail: arobles@ugr.es

${ }^{\ddagger}$ Departamento de Álgebra, Universidad de Granada, 18071-Granada, Spain.

E-mail: jrosales@ugr.es
} 
through an example (in fact, an observation attributed to J. Backelin), it was proved that there does not exist an upper bound for the type when the embedding dimension is greater than four.

The reader unfamiliar with the theory of numerical semigroups may find that the terminology used (embedding dimension, genus, type) is a bit strange. However, let us observe that there are many works dedicated to the studying of analytically irreducible one dimension local domains via their value semigroups, which precisely are numerical semigroups. All these invariants (see [2]) have an interpretation in this theory. This shows that numerical semigroups with type equal to one are very interesting (see [12]). Such numerical semigroups are called symmetric.

It is clear that $\mathrm{F}(S) \in \operatorname{PF}(S)$. Thus, the type of a numerical semigroup $S$ is one if and only if $\operatorname{PF}(S)=$ $\{\mathrm{F}(S)\}$. On the other hand, as a consequence of [8, Lemma 1], we know that a numerical semigroup $S$ is symmetric if and only if $\mathrm{g}(S)=\frac{\mathrm{F}(S)+1}{2}$. Therefore, the Frobenius number of a symmetric numerical semigroup is always an odd integer and, moreover, all numerical semigroups of type one and the same Frobenius number have the same genus. By applying the above characterization, it is easy to check that, if $F$ is a non-negative odd integer, then $\left\{0, \frac{F+1}{2}, \rightarrow\right\} \backslash\{F\}$ is a symmetric numerical semigroup with Frobenius number $F$ (where the symbol $\rightarrow$ means that every integer greater than $\frac{F+1}{2}$ belongs to the set). Consequently, we can assert that $\{F\}$ is the set of pseudo-Frobenius numbers of a numerical semigroup of type one if and only if $F$ is an odd integer greater than or equal to -1 . (Let us observe that $\mathbb{N}$ is a symmetric numerical semigroup with Frobenius number -1.)

The above paragraph leads us to propose the following questions which arise naturally for numerical semigroups of type two.

- Question 1: What conditions must two integers $g_{1}, g_{2}$ satisfy in order to there exist a numerical semigroup $S$ such that $\operatorname{PF}(S)=\left\{g_{1}, g_{2}\right\}$ ?

- Question 2: If two numerical semigroups of type two have the same Frobenius number, what are their genera?

- Question 3: If two numerical semigroups of type two have the same set of pseudo-Frobenius numbers, what are their genera?

Our purpose in this work is to answer these questions. Concretely, we will show the following results.

Theorem 2.10. Let $g_{1}, g_{2}$ be two positive integers such that $g_{1}<g_{2}$ and let

$$
\theta\left(g_{1}, g_{2}\right)=\left\{\begin{array}{cl}
2 g_{1}-g_{2}, & \text { if } g_{2} \text { is odd }, \\
g_{1}-\frac{g_{2}}{2}, & \text { if } g_{2} \text { is even. }
\end{array}\right.
$$

Then there exists a numerical semigroup $S$ such that $\operatorname{PF}(S)=\left\{g_{1}, g_{2}\right\}$ if and only if $\frac{g_{2}}{2}<g_{1}$ and $\theta\left(g_{1}, g_{2}\right)$ does not divide any element of the set $\left\{g_{1}, g_{2}, g_{2}-g_{1}\right\}$.

It is clear that Theorem 2.10 is the answer to Question 1.

Theorem 3.3. If $S$ is a numerical semigroup of type two, then

$$
\frac{\mathrm{F}(S)+2}{2} \leq \mathrm{g}(S) \leq \frac{2(\mathrm{~F}(S)+1)}{3} .
$$

In order to answer Question 2, we will complement Theorem 3.3 by showing that, if $F+1$ is a positive integer divisible by six, then, for each integer $n \in\left[\frac{F+2}{2}, \frac{2(F+1)}{3}\right]$, there exists a numerical semigroup $S$ of type two such that $\mathrm{F}(S)=F$ and $\mathrm{g}(S)=n$.

If $q$ is a rational number, then we will denote by $\lceil q\rceil$ and $\lfloor q\rfloor$ the numbers $\min \{z \in \mathbb{Z} \mid q \leq z\}$ and $\max \{z \in \mathbb{Z} \mid z \leq q\}$, respectively. As a consequence of Theorem 3.3 , we know that, if $S$ is a numerical semigroup of type two, then $\left\lceil\frac{\mathrm{F}(S)+2}{2}\right\rceil \leq \mathrm{g}(S) \leq\left\lfloor\frac{2(\mathrm{~F}(S)+1)}{3}\right\rfloor$.

Theorem 4.6. If $k \in \mathbb{N} \backslash\{0\}$ and $F=6 k-1$, then there exist two numerical semigroups, $S$ and $S^{\prime}$, such that $\operatorname{PF}(S)=\operatorname{PF}\left(S^{\prime}\right)=\{F-1, F\}, \mathrm{g}(S)=\left\lfloor\frac{2(F+1)}{3}\right\rfloor$ and $\mathrm{g}\left(S^{\prime}\right)=\left\lceil\frac{F+2}{2}\right\rceil$.

Let us observe that Theorem 4.6 answers Question 3 and also shows that the bounds of Theorem 3.3 are achieved in numerical semigroups of type two that have the same set of pseudo-Frobenius numbers. 
As might be expected, we will state these three theorems in three different sections (Section 2, 3 , and 4 , respectively). In addition, in Section 5 we will show that in some cases all values, between the two bounds given in Theorem 3.3 , are suitable as the genus of a numerical semigroup of type two.

Finally, we point out that, in order to make it easier to locate some results and facts, together with the original references, we direct the reader to [21], in which the corresponding references are given.

\section{Sets of pseudo-Frobenius numbers of numerical semigroups of type two}

In this section we are interested in showing the conditions that two integers have to satisfy in order to be the pseudo-Frobenius numbers of a numerical semigroup of type two.

It is easy to see that, if $S$ is a numerical semigroup different from $\mathbb{N}$, then $\operatorname{PF}(S) \subseteq \mathbb{N} \backslash\{0\}$. Therefore, the first condition is that $g_{1}, g_{2}$ must be positive integers.

Before considering the general case, we study a particular one for which we can give a quick answer. A numerical semigroup is called pseudo-symmetric if $\operatorname{PF}(S)=\left\{\frac{\mathrm{F}(S)}{2}, \mathrm{~F}(S)\right\}$ (see Corollary 4.16 in [21]). This class of numerical semigroups has also been widely studied and likewise there are many characterizations of them. For instance (see Corollary 4.5 in [21]), $S$ is a pseudo-symmetric numerical semigroup if and only if $\mathrm{g}(S)=\frac{\mathrm{F}(S)+2}{2}$. Thus, the Frobenius number of a pseudo-symmetric numerical semigroup is always an even positive integer. Moreover, applying the characterization, we can state that, if $F$ is an even positive integer, then $\left\{0, \frac{F}{2}+1, \rightarrow\right\} \backslash\{F\}$ is a pseudo-symmetric numerical semigroup with Frobenius number $F$. Therefore, if $g_{2}$ is an even positive integer, then there always exists a numerical semigroup $S$ such that $\operatorname{PF}(S)=\left\{\frac{g_{2}}{2}, g_{2}\right\}$.

From now on, we suppose that $g_{1}, g_{2}$ are two positive integers such that $g_{1}<g_{2}$ and $g_{1} \neq \frac{g_{2}}{2}$.

Lemma 2.1. Let $S$ be a numerical semigroup such that $\operatorname{PF}(S)=\left\{g_{1}, g_{2}\right\}$. Then

$$
g_{1}=\max \left\{x \in \mathbb{N} \backslash S \mid x \neq \frac{g_{2}}{2} \text { and } g_{2}-x \notin S\right\} .
$$

Proof. First of all, taking $A=\left\{x \in \mathbb{N} \backslash S \mid x \neq \frac{g_{2}}{2}\right.$ and $\left.g_{2}-x \notin S\right\}$, we see that there exists $h=\max \{A\}$. Since $g_{1} \in \operatorname{PF}(S)$, if $g_{2}-g_{1} \in S$, then $g_{2}=g_{1}+\left(g_{2}-g_{1}\right) \in S$, which is a contradiction. Moreover, by the considered conditions, $g_{1} \neq \frac{g_{2}}{2}$. Thereby, $g_{1} \in A$. The existence of $h$ follows from the inclusion $A \subseteq \mathbb{N} \backslash S$.

Now, let us observe that, if $x \in A$, then $g_{2}-x \in A$. Therefore, $h>\frac{g_{2}}{2}$.

Finally, if $h \notin \operatorname{PF}(S)$, then there exists $s \in S \backslash\{0\}$ such that $h+s \notin S$ and $h+s>h>\frac{g_{2}}{2}$. Moreover, if $g_{2}-(h+s) \in S$, then $g_{2}-h=\left(g_{2}-(h+s)\right)+s \in S$ that is not possible. Thus, $h+s \in A$, in contradiction with the definition of $h$. Therefore, $h \in \operatorname{PF}(S)$. Since $g_{2} \notin A$, we conclude that $h=g_{1}$.

From the proof of the previous lemma, we have the following result.

Lemma 2.2. Let $S$ be a numerical semigroup such that $\operatorname{PF}(S)=\left\{g_{1}, g_{2}\right\}$. Then $\frac{g_{2}}{2}<g_{1}$.

The next result is an immediate consequence of Proposition 2.19 in 21].

Lemma 2.3. Let $S$ be a numerical semigroup and let $x \in \mathbb{N} \backslash S$. Then there exists $g \in \operatorname{PF}(S)$ such that $g-x \in S$.

The following lemma gives us two essential elements of $S$ and a particular element of $\mathbb{N} \backslash S$ which are necessary to know in order to achieve our aim.

Lemma 2.4. Let $S$ be a numerical semigroup such that $\operatorname{PF}(S)=\left\{g_{1}, g_{2}\right\}$. Then:

(i) $g_{2}-g_{1} \notin S$.

(ii) $2 g_{1}-g_{2} \in S$.

(iii) If $g_{2}$ is even, then $g_{1}-\frac{g_{2}}{2} \in S$.

Proof. (i) See the proof of Lemma 2.1

(ii) Since $g_{2}-g_{1} \notin S$, applying Lemma 2.3 , we have that $g_{2}-\left(g_{2}-g_{1}\right) \in S$ or $g_{1}-\left(g_{2}-g_{1}\right) \in S$. The first option is not possible and, therefore, $2 g_{1}-g_{2} \in S$. 
(iii) Since $g_{2} \notin S$, then $\frac{g_{2}}{2} \notin S$. Applying again Lemma 2.3 we have that $g_{2}-\frac{g_{2}}{2} \in S$ or $g_{1}-\frac{g_{2}}{2} \in S$. Once again the first option is not possible and, thereby, we get that $g_{1}-\frac{g_{2}}{2} \in S$.

The next result gives us the condition that two integers $g_{1}, g_{2}$ have to satisfy, if $g_{2}$ is odd, in order to get a numerical semigroup $S$ such that $\operatorname{PF}(S)=\left\{g_{1}, g_{2}\right\}$. As usual, if $a, b$ are positive integers, we denote by $a \mid b$ that $a$ divides $b$. When $b$ is not divisible by $a$, we write $a \nmid b$. On the other hand, if $a, b, c$ are integers such that $c \neq 0$, then $a \equiv b(\bmod c)$ means that $c \mid(a-b)$.

Proposition 2.5. Let $g_{1}, g_{2}$ be two positive integers such that $g_{1}<g_{2}$ and $g_{2}$ is odd. Then there exists a numerical semigroup $S$ such that $\mathrm{PF}(S)=\left\{g_{1}, g_{2}\right\}$ if and only if $\frac{g_{2}}{2}<g_{1},\left(2 g_{1}-g_{2}\right) \nmid g_{1},\left(2 g_{1}-g_{2}\right) \nmid g_{2}$, and $\left(2 g_{1}-g_{2}\right) \nmid\left(g_{2}-g_{1}\right)$.

Proof. (Necessity) By Lemma 2.2, we know that $\frac{g_{2}}{2}<g_{1}$. On the other hand, by Lemma $2.42 g_{1}-g_{2} \in S$ and $g_{2}-g_{1} \notin S$. Since $g_{1}, g_{2} \notin S$, we have that $\left(2 g_{1}-g_{2}\right) \nmid g_{1},\left(2 g_{1}-g_{2}\right) \nmid g_{2}$, and $\left(2 g_{1}-g_{2}\right) \nmid\left(g_{2}-g_{1}\right)$.

(Sufficiency) Let us take $S=\left(\langle m\rangle \cup\left\{\frac{g_{2}+1}{2}, \rightarrow\right\}\right) \backslash\left(\mathrm{R}\left(g_{1}\right) \cup \mathrm{R}\left(g_{2}\right)\right)$, with $m=2 g_{1}-g_{2}$ and, for each $i \in\{1,2\}, \mathrm{R}\left(g_{i}\right)=\left\{g_{i}-k m \mid k \in \mathbb{N}\right\}$. By straightforward computations, we have that $S$ is a numerical semigroup with $\left\{g_{1}, g_{2}\right\} \subseteq \operatorname{PF}(S)$. Therefore, we will finish the proof if we show that, if $x \in \mathbb{N} \backslash\left(S \cup\left\{g_{1}, g_{2}\right\}\right)$, then there exists $s \in S$ such that $x+s \notin S$.

If $x \equiv g_{i}(\bmod m)$ for some $i \in\{1,2\}$, then $x=g_{i}-k m$ for some $k \in \mathbb{N} \backslash\{0\}$. Therefore, $k m \in S \backslash\{0\}$ and $x+k m=g_{i} \notin S$. Thus, from now on, we can assume that $x \not \equiv g_{i}(\bmod m)$ for all $i \in\{1,2\}$.

Since $x \notin S$, we have that $x<\frac{g_{2}+1}{2}$ and, thereby, $g_{2}-x \geq \frac{g_{2}+1}{2}$. If $g_{2}-x \in S$ then $g_{2}-x \in S \backslash\{0\}$ and $x+\left(g_{2}-x\right)=g_{2} \notin S$. Now, let us see that the case $g_{2}-x \notin S$ is not possible. Indeed, if $g_{2}-x \notin S$, then $g_{2}-x \in \mathrm{R}\left(g_{2}\right)$ or $g_{2}-x \in \mathrm{R}\left(g_{1}\right)$ because $g_{2}-x \geq \frac{g_{2}+1}{2}$. On the one hand, if $g_{2}-x \in \mathrm{R}\left(g_{2}\right)$, then $g_{2}-x=g_{2}-k m$ for some $k \in \mathbb{N}$ and so $x=k m \in S$, which is a contradiction. On the other hand, if $g_{2}-x \in \mathrm{R}\left(g_{1}\right)$, then $g_{2}-x=g_{1}-k m$ for some $k \in \mathbb{N}$. Thus, $x=g_{2}-\left(g_{1}-k m\right)=\left(g_{2}-g_{1}+m\right)+(k-1) m=g_{1}+(k-1) m$, which contradicts $x \not \equiv g_{1}(\bmod m)$.

Remark 2.6. We have considered the sets $\mathrm{R}\left(g_{1}\right)$ and $\mathrm{R}\left(g_{2}\right)$ to make explicit that we remove all the possible elements of $S$ in order to have $g_{1}, g_{2} \notin S$. In fact, it is sufficient to consider $\left\{g_{1}\right\},\left\{g_{2}-k m \mid k \in\left\{0,1, \ldots,\left\lfloor\frac{g_{2}-1}{2 m}\right\rfloor\right\}\right\}$ instead of $\mathrm{R}\left(g_{1}\right), \mathrm{R}\left(g_{2}\right)$, respectively.

We illustrate Proposition 2.5 with an example.

Example 2.7. We are going to build a numerical semigroup $S$ such that $\operatorname{PF}(S)=\{7,9\}$. Since $\frac{9}{2}<7$, $5 \nmid 7,5 \nmid 9$, and $5 \nmid 2$, by Proposition 2.5, we deduce that there exists at least one such numerical semigroup. Moreover, from the proof of sufficiency, we know that $S=(\langle 5\rangle \cup\{5, \rightarrow\}) \backslash(\mathrm{R}(7) \cup \mathrm{R}(9))=\{0,5,6,8,10, \rightarrow$ \}$=\langle 5,6,8\rangle$ is a numerical semigroup with $\operatorname{PF}(S)=\{7,9\}$.

The following result gives us the condition that two integers $g_{1}, g_{2}$ have to satisfy, if $g_{2}$ is even, in order to have a numerical semigroup $S$ such that $\mathrm{PF}(S)=\left\{g_{1}, g_{2}\right\}$.

Proposition 2.8. Let $g_{1}, g_{2}$ be two positive integers such that $g_{1}<g_{2}$ and $g_{2}$ is even. Then there exists a numerical semigroup $S$ such that $\operatorname{PF}(S)=\left\{g_{1}, g_{2}\right\}$ if and only if $\frac{g_{2}}{2}<g_{1},\left(g_{1}-\frac{g_{2}}{2}\right) \nmid g_{1},\left(g_{1}-\frac{g_{2}}{2}\right) \nmid g_{2}$, and $\left(g_{1}-\frac{g_{2}}{2}\right) \nmid\left(g_{2}-g_{1}\right)$.

Proof. Taking the set $S=\left(\langle m\rangle \cup\left\{\frac{g_{2}}{2}+1, \rightarrow\right\}\right) \backslash\left(\mathrm{R}\left(g_{1}\right) \cup \mathrm{R}\left(g_{2}\right)\right)$ with $m=g_{1}-\frac{g_{2}}{2}$, the proof of this proposition follows the same ideas of the proof for Proposition 2.5. Therefore, we omit it.

Let us illustrate Proposition 2.8 with an example.

Example 2.9. We are going to build a numerical semigroup $S$ such that $\operatorname{PF}(S)=\{9,10\}$. Since $5<9$, $4 \nmid 9,4 \nmid 10$, and $4 \nmid 1$, by Proposition 2.8 , we deduce that there exists at least one such numerical semigroup. Moreover, from the proof of sufficiency, we get that $S=(\langle 4\rangle \cup\{6, \rightarrow\}) \backslash(\mathrm{R}(9) \cup \mathrm{R}(10))=\{0,4,7,8,11, \rightarrow$ \}$=\langle 4,7,13\rangle$ is a numerical semigroup with $\operatorname{PF}(S)=\{9,10\}$.

We finish this section with the combination of Propositions 2.5 and 2.8 in order to obtain the first of the main results in this paper and so answer the first question.

Theorem 2.10. Let $g_{1}, g_{2}$ be two positive integers such that $g_{1}<g_{2}$ and let

$$
\theta\left(g_{1}, g_{2}\right)=\left\{\begin{array}{l}
2 g_{1}-g_{2}, \text { if } g_{2} \text { is odd, } \\
g_{1}-\frac{g_{2}}{2}, \text { if } g_{2} \text { is even. }
\end{array}\right.
$$

Then there exists a numerical semigroup $S$ such that $\operatorname{PF}(S)=\left\{g_{1}, g_{2}\right\}$ if and only if $\frac{g_{2}}{2}<g_{1}$ and $\theta\left(g_{1}, g_{2}\right)$ does not divides any element of the set $\left\{g_{1}, g_{2}, g_{2}-g_{1}\right\}$. 


\section{Bounding the genus by the Frobenius number}

If $S$ is a numerical semigroup we denote by $\mathrm{n}(S)$ the cardinality of the set $\{s \in S \mid s<\mathrm{F}(S)\}$. Observe that $\mathrm{g}(S)+\mathrm{n}(S)=\mathrm{F}(S)+1$. The next result is easily deduced from Theorem 20 in [8] (alternatively see Proposition 2.26 in 21]).

Lemma 3.1. If $S$ is a numerical semigroup, then $\mathrm{g}(S) \leq \mathrm{t}(S) \mathrm{n}(S)$.

The following result is a direct consequence of Lemma 1 and Proposition 2 in $[8$, (or see Lemma 2.14 and Corollary 4.5 in [21]).

Lemma 3.2. If $S$ is a numerical semigroup, then $\mathrm{g}(S) \geq \frac{\mathrm{F}(S)+1}{2}$. Moreover, $\mathrm{g}(S)=\frac{\mathrm{F}(S)+1}{2}$ if and only if $\mathrm{t}(S)=1$.

Now, we can bound the genus of a numerical semigroup of type two by its Frobenius number.

Theorem 3.3. If $S$ is a numerical semigroup of type two, then

$$
\frac{\mathrm{F}(S)+2}{2} \leq \mathrm{g}(S) \leq \frac{2(\mathrm{~F}(S)+1)}{3} .
$$

Proof. By Lemma 3.2 , we have that $\mathrm{g}(S) \geq \frac{\mathrm{F}(S)+2}{2}$ if $\mathrm{F}(S)$ is even and that $\mathrm{g}(S) \geq \frac{\mathrm{F}(S)+3}{2}$ if $\mathrm{F}(S)$ is odd. Thus, $\mathrm{g}(S) \geq \frac{\mathrm{F}(S)+2}{2}$.

On the other hand, by Lemma 3.1 we know that $\mathrm{g}(S) \leq 2 \mathrm{n}(S)$. Thereby, $3 \mathrm{~g}(S) \leq 2 \mathrm{n}(S)+2 \mathrm{~g}(S)=$ $2(\mathrm{~F}(S)+1)$.

Our next aim will be to show that the given bounds in the previous theorem are reached. First of all, from Section 2 we have that, if $F$ is an even positive integer, then $S=\left\{0, \frac{F}{2}+1, \rightarrow\right\} \backslash\{F\}$ is a (pseudo-symmetric) numerical semigroup with Frobenius number $F$, type two and $\mathrm{g}(S)=\frac{\mathrm{F}(S)+2}{2}$. Moreover, $\operatorname{PF}(S)=\left\{F, \frac{F}{2}\right\}$.

Now we will see that there exists a numerical semigroup $S$ of type two such that $\mathrm{g}(S)=\frac{2(\mathrm{~F}(S)+1)}{3}$. First, we need the following result that is an immediate consequence of Theorems 11 and 14 in $[8$ (alternatively see Proposition 10.21 in [21]).

Lemma 3.4. If $S$ is a numerical semigroup with $\mathrm{e}(S)=3$ and pairwise relatively prime minimal generators, then $\mathrm{t}(S)=2$.

We are ready to prove the result announced just before lemma 3.4 .

Proposition 3.5. If $k \in \mathbb{N} \backslash\{0\}$, then $S=\langle 3,3 k+1,3 k+2\rangle$ is a numerical semigroup of type two with $\mathrm{g}(S)=\frac{2(\mathrm{~F}(S)+1)}{3}$. Moreover, $\operatorname{PF}(S)=\{\mathrm{F}(S), \mathrm{F}(S)-1\}=\{3 k-1,3 k-2\}$.

Proof. As an immediate consequence of Lemma 3.4 we have that $S=\langle 3,3 k+1,3 k+2\rangle$ is a numerical semigroup of type two. Also, it is clear that $\operatorname{PF}(S)=\{3 k-1,3 k-2\}$. Finally, let us observe that $\mathbb{N} \backslash S=$ $\{1,2, \ldots, 3 k-1\} \backslash\{1 \cdot 3,2 \cdot 3, \ldots,(k-1) \cdot 3\}$. Thereby, $\mathrm{g}(S)=3 k-1-(k-1)=2 k$ and, therefore, $\frac{2(\mathrm{~F}(S)+1)}{3}=\frac{2 \cdot 3 k}{3}=2 k=\mathrm{g}(S)$.

We finish this section with an easy result which can be considered as a first answer to Question 2. Indeed, we get numerical semigroups of type two with the same Frobenius number and different genus.

Corollary 3.6. Let $k$ be a positive integer. Then

(i) $S=\{0,3 k-1, \rightarrow\} \backslash\{6 k-4\}$ is a (pseudo-symmetric) numerical semigroup of type two, $\mathrm{g}(S)=3 k-1$, and $\operatorname{PF}(S)=\{3 k-2,6 k-4\}$.

(ii) $S=\langle 3,6 k-2,6 k-1\rangle$ is a numerical semigroup of type two, $\mathrm{g}(S)=4 k-2$, and $\mathrm{PF}(S)=\{6 k-5,6 k-4\}$. 


\section{Numerical semigroups with the same set of pseudo-Frobenius numbers}

As a consequence of Theorem 3.3 if $S$ is a numerical semigroup of type two, then we know that $\left\lceil\frac{\mathrm{F}(S)+2}{2}\right\rceil \leq$ $\mathrm{g}(S) \leq\left\lfloor\frac{2(\mathrm{~F}(S)+1)}{3}\right\rfloor$. Our aim in this section will be to prove that, if $k \in \mathbb{N} \backslash\{0\}$ and $F=6 k-1$, then there exist two numerical semigroups $S$ and $S^{\prime}$ such that $\operatorname{PF}(S)=\operatorname{PF}\left(S^{\prime}\right)=\{F, F-1\}, \mathrm{g}(S)=\left\lfloor\frac{2(F+1)}{3}\right\rfloor$, and $\mathrm{g}\left(S^{\prime}\right)=\left\lceil\frac{F+2}{2}\right\rceil$. This fact shows that the bounds given in Theorem 3.3 are reached for numerical semigroups with the same set of pseudo-Frobenius numbers.

The next result was established in the introduction.

Lemma 4.1. If $F$ is an odd positive integer, then $S=\left\{0, \frac{F+1}{2}, \rightarrow\right\} \backslash\{F\}$ is a numerical semigroup of type one (that is, a symmetric numerical semigroup) with Frobenius number $F$.

It is clear that, if $S$ is a numerical semigroup and $x$ is a minimal generator of $S$, then $S \backslash\{x\}$ is also a numerical semigroup. The following result is Lemma 24 of [19].

Lemma 4.2. If $S$ is a numerical semigroup of type one and $x$ is a minimal generator of $S$ such that $x<\mathrm{F}(S)$, then $\operatorname{PF}(S \backslash\{x\})=\{\mathrm{F}(S), x\}$ if and only if $2 x-\mathrm{F}(S) \in S$.

Let us observe that, if $S$ is a numerical semigroup of type two and $\mathrm{F}(S)$ is odd, then $\mathrm{F}(S) \geq 5$ because:

1. if $\mathrm{F}(S)=-1$, then $S=\mathbb{N}$ with $\mathrm{t}(\mathbb{N})=1$;

2. if $\mathrm{F}(S)=1$, then $S=\langle 2,3\rangle$ with $\mathrm{t}(\langle 2,3\rangle)=1$;

3. if $\mathrm{F}(S)=3$, then $S=\langle 2,5\rangle$ with $\mathrm{t}(\langle 2,5\rangle)=1$ or $S=\langle 4,5,6,7\rangle$ with $\mathrm{t}(\langle 4,5,6,7\rangle)=3$.

Proposition 4.3. If $F$ is an odd integer greater than or equal to five, then $S=\left\{0, \frac{F+1}{2}, \rightarrow\right\} \backslash\{F, F-1\}$ is a numerical semigroup of type two with $\mathrm{F}(S)=F, \mathrm{~g}(S)=\frac{\mathrm{F}(S)+3}{2}=\left\lceil\frac{\mathrm{F}(S)+2}{2}\right\rceil$, and $\operatorname{PF}(S)=\{F, F-1\}$.

Proof. Let $S^{\prime}=\left\{0, \frac{F+1}{2}, \rightarrow\right\} \backslash\{F\}$. By Lemma 4.1, we know that $S^{\prime}$ is a numerical semigroup of type one with Frobenius number $F$. Moreover, it is clear that $F-1$ is a minimal generator of $S^{\prime}$ less than $F$. We claim that $2(F-1)-F \in S^{\prime}$. Indeed, since $F \geq 5$, then $2(F-1)-F=F-2$ is an integer greater than or equal to $\frac{F+1}{2}$ and different from $F$. Thus, we have the assertion.

Now, since $S=S^{\prime} \backslash\{F-1\}$, applying Lemma 4.2 we get that $S$ is a numerical semigroup of type two and $\operatorname{PF}(S)=\{F, F-1\}$. And, since $\mathrm{t}\left(S^{\prime}\right)=1$, applying Lemma 3.2 we have that $\mathrm{g}\left(S^{\prime}\right)=\frac{F+1}{2}$. Finally, it is clear that $\mathrm{F}(S)=F$ and, since $\mathrm{g}(S)=\mathrm{g}\left(S^{\prime}\right)+1$, that $\mathrm{g}(S)=\frac{\mathrm{F}(S)+3}{2}$.

Remark 4.4. Let us take a positive integer $k$ and consider $F=6 k-1$. As a consequence of Theorem 3.3 , we have that, if $S$ is a numerical semigroup of type two with Frobenius number $F$, then $\frac{F+3}{2} \leq \mathrm{g}(S) \leq \frac{2(F+1)}{3}$ and, therefore, $3 k+1 \leq \mathrm{g}(S) \leq 4 k$.

This remark leads us to the next result.

Corollary 4.5. If $k$ is a positive integer, then $S=\langle 3,6 k+1,6 k+2\rangle$ and $S^{\prime}=\{0,3 k, \rightarrow\} \backslash\{6 k-1,6 k-2\}$ are two numerical semigroups with $\mathrm{PF}(S)=\operatorname{PF}\left(S^{\prime}\right)=\{6 k-1,6 k-2\}$. Moreover, $\mathrm{g}(S)=4 k$ and $\mathrm{g}\left(S^{\prime}\right)=3 k+1$.

Proof. Let $k^{\prime}=2 k$. Then $S=\left\langle 3,3 k^{\prime}+1,3 k^{\prime}+2\right\rangle$ and, by Proposition 3.5 , we know that $S$ is a numerical semigroup of type two with $\mathrm{F}(S)=3 k^{\prime}-1=6 k-1, \mathrm{~g}(S)=\frac{2 \cdot 6 k}{3}=4 k$, and $\mathrm{PF}(S)=\{6 k-1,6 k-2\}$.

Now, let $F=6 k-1$. It is clear that $F$ is an odd integer greater than or equal to 5 and that $S^{\prime}=$ $\left\{0, \frac{F+1}{2}, \rightarrow\right\} \backslash\{F, F-1\}$. Therefore, applying Proposition 4.3 , we get that $S^{\prime}$ is a numerical semigroup of type two with $\mathrm{F}(S)=F=6 k-1, \mathrm{~g}(S)=\frac{F+3}{2}=3 k+1$, and $\mathrm{PF}(S)=\{6 k-1,6 k-2\}$.

The above corollary shows that two numerical semigroups of type two with the same set of pseudoFrobenius numbers can have different genus. Furthermore, it shows that the bounds of theorem 3.3 are achieved for numerical semigroups with the same set of pseudo-Frobenius numbers, as we noted at the beginning of this section. Thus, we can establish the following result.

Theorem 4.6. If $k \in \mathbb{N} \backslash\{0\}$ and $F=6 k-1$, then there exist two numerical semigroups, $S$ and $S^{\prime}$, such that $\mathrm{PF}(S)=\mathrm{PF}\left(S^{\prime}\right)=\{F-1, F\}, \mathrm{g}(S)=\left\lfloor\frac{2(F+1)}{3}\right\rfloor$ and $\mathrm{g}\left(S^{\prime}\right)=\left\lceil\frac{F+2}{2}\right\rceil$. 


\section{Possible values of the genus}

In order to finish this work we will see that, for each $n \in\{3 k+1, \ldots, 4 k\}$, there exists a numerical semigroup $S$ of type two such that $\mathrm{F}(S)=6 k-1$ and $\mathrm{g}(S)=n$. This fact, together with Remark 4.4, shows that all values, between the two bounds given in Theorem 3.3 , are suitable as a genus of a numerical semigroup of type two.

Let $S$ be a numerical semigroup and $s \in S \backslash\{0\}$. The Apéry set of $s$ in $S$ (see [1]) is $\operatorname{Ap}(S, s)=\{x \in S \mid$ $x-s \notin S\}$. It is easy to check that $\operatorname{Ap}(S, s)=\{w(0)=0, w(1), \ldots, w(s-1)\}$, where $w(i)$ is the least element of $S$ congruent with $i$ modulo $s$, for all $i \in\{0,1, \ldots, s-1\}$.

The next result is consequence of Lemma 3 in [4] and the Theorem in [22, page 3] (or see Proposition 2.12 in [21]).

Lemma 5.1. Let $S$ be a numerical semigroup and let $s$ be a non-zero element of $S$. Then

(i) $\mathrm{F}(S)=\max (\mathrm{Ap}(S, s))-s$,

(ii) $\mathrm{g}(S)=\frac{1}{s}\left(\sum_{w \in \operatorname{Ap}(S, s)} w\right)-\frac{s-1}{2}$.

With this lemma, we are ready to prove the result mentioned at the beginning of this section.

Theorem 5.2. Let $k$ be a positive integer, let $n \in\{3 k+1, \ldots, 4 k\}$, and let $r=4 k-n$. Then $S=$ $\langle 3,6 k+1-3 r, 6 k+2\rangle$ is a numerical semigroup of type two such that $\mathrm{F}(S)=6 k-1$ and $\mathrm{g}(S)=n$.

Proof. First of all, let us see that $\{3,6 k+1-3 r, 6 k+2\}$ is a minimal system of generators of $S$. To do this, since $6 k+1-3 r<6 k+2,(6 k+1-3 r) \equiv 1(\bmod 3)$, and $(6 k+2) \equiv 2(\bmod 3)$, it is enough to show that $2(6 k+1-3 r)>6 k+2$. In fact, $2(6 k+1-3 r)>6 k+2$ if and only if $r<k$, that it is true because $r=4 k-n \leq 4 k-(3 k+1)=k-1$.

As an immediate consequence of the previous paragraph, we have that $\operatorname{Ap}(S, 3)=\{0,6 k+1-3 r, 6 k+2\}$. Therefore, applying Lemma 5.1. we get that $\mathrm{F}(S)=6 k+2-3=6 k-1$ and $\mathrm{g}(S)=\frac{1}{3}(6 k+1-3 r+6 k+2)-\frac{3-1}{2}=$ $n$.

Finally, from Lemma 3.4 , we have that $S$ is a numerical semigroup of type two.

Let us observe that, as a consequence of Proposition 2.20 in [21, we conclude that $\operatorname{PF}(S)=\{6 k+1-$ $3(r-1), 6 k-1\}$, where $S$ is the numerical semigroup in the preceding proposition.

\section{References}

[1] R. Apéry. Sur les branches superlinéaires des courbes algébriques. C. R. Acad. Sci. Paris 222 (1946), $1198-1200$.

[2] V. Barucci, D. E. Dobbs, and M. Fontana. Maximality Properties in Numerical Semigroups and Applications to One-Dimensional Analytically Irreducible Local Domains. Mem. Amer. Math. Soc., vol. 125 (no. 598) (Providence: American Mathematical Society, 1997).

[3] A. Brauer. On a problem of partitions. Amer. J. Math. 64 (1942), 299-312.

[4] A. Brauer, J. E. Shockley. On a problem of Frobenius. J. Reine Angew. Math. 211 (1962), 215-220.

[5] F. Curtis. On formulas for the Frobenius number of a numerical semigroup. Math. Scand. 67 (1990), $190-192$.

[6] J. L. Davison. On the linear Diophantine problem of Frobenius. J. Number Theory 48 (1994), 353-363.

[7] M. Djawadi, G. Hofmeister. Linear Diophantine problems. Arch. Math. (Basel) 66 (1996), 19-29.

[8] R. Fröberg, G. Gottlieb, and R. Häggkvist. On numerical semigroups. Semigroup Forum 35 (1987), 63-83.

[9] H. Greenberg. Solution to a Diophantine equation for nonnegative integers. J. Algorithms 9 (1988), 343353.

[10] S. M. Johnson. A linear Diophantine problem. Canad. J. Math. 12 (1960), 390-398. 
[11] R. Kannan. Lattice translates of a polytope and the Frobenius problem. Combinatorica 12 (1992), 161177.

[12] E. Kunz. The value-semigroup of a one-dimensional Gorenstein ring. Proc. Amer. Math. Soc. 25 (1970), $748-751$.

[13] J. L. Ramírez Alfonsín. Complexity of the Frobenius problem. Combinatorica 16 (1996), 143-147.

[14] J. L. Ramírez Alfonsín. The Diophantine Frobenius problem. Oxford Lecture Series in Mathematics and its Applications, vol. 30 (Oxford: Oxford Univ. Press, 2005).

[15] J. L. Ramírez Alfonsín and Ø. J. Rødseth. Numerical semigroups: Apéry sets and Hilbert series. Semigroup Forum 79 (2009), 323-340.

[16] A.M. Robles-Pérez and J.C. Rosales. The Frobenius problem for numerical semigroups with embedding dimension equal to three. Math. Comput. 81 (2012), 1609-1617.

[17] Ö. J. Rödseth. On a linear Diophantine problem of Frobenius. J. Reine Angew. Math. 301 (1978), 171178.

[18] Ö. J. Rödseth. On a linear Diophantine problem of Frobenius II. J. Reine Angew. Math. 307/308 (1979), 431-440.

[19] J. C. Rosales. Numerical semigroups that differ from a symmetric numerical semigroup in one element. Algebra Colloq. 15 (2008), 23-32.

[20] J. C. Rosales and M. B. Branco. Numerical semigroups that can be expressed as an intersection of symmetric numerical semigroups. J. Pure Appl. Algebra 171 (2002), 303-314.

[21] J. C. Rosales and P. A. García-Sánchez. Numerical semigroups. Developments in Mathematics, vol. 20 (New York: Springer, 2009).

[22] E. S. Selmer. On the linear Diophantine problem of Frobenius. J. Reine Angew. Math. 293/294 (1977), $1-17$.

[23] J. J. Sylvester. On subvariants, i.e. semi-invariants to binary quantics of an unlimited order. Amer. J. Math. 5 (1882), 79-136.

[24] J. J. Sylvester. Problem 7382. The Educational Times, and Journal of the College of Preceptors, New Series, 36(266) (1883), 177. Solution by W. J. Curran Sharp, ibid., 36(271) (1883), 315. Republished as [25.

[25] J. J. Sylvester. Problem 7382. In W. J. C. Miller (Ed.), Mathematical questions, with their solutions, from The Educational Times, vol. 41, page 21 (Francis Hodgson, London, 1884). 